What is the function of the poly(A) sequence? Is it related solely to the maturation process, perhaps because it is needed for nucleocytoplasmic transport of mRNA, or does it serve some function in the cytoplasm? That its role is not solely nuclear has been inferred from experiments which demonstrated the presence of poly(A) in viral RNAs which function solely in the cytoplasm. Support for the view that poly(A) plays some part essential to the function of all mRNAs in eukaryotic cells is provided also by the observation that the messengers of mitochondria possess a short length of poly (A).

Mitochondria possess a protein synthetic system which differs in several ways from that of the surrounding cytoplasm, in some ways showing analogies to bacterial protein synthesis. The presence of poly (A) sequences has been used to isolate a mitochondrial RNA fraction which seems to represent the messengers; their length of poly(A) is fifty-six bases, somewhat shorter than

\section{Solar neutrino problem still unsolved}

A Few months ago, Prentice suggested that the absence of neutrinos from the Sun could be explained if that star has a hydrogen-free core amounting to about 0.02 or 0.03 $M_{\odot}$ (Mon. Not. R. astr. Soc., 163, $331 ; 1973)$. Such helium inhomogeneities reduced the neutrino flux by a factor of 10 , or least for the simple polytropic models used by Prentice. But now more detailed calculations by Demarque et al. (Mon. Not. R. astr. Soc., 165, 19P; 1973) suggest that this is not the case for realistic solar models.

These detailed models confirm Prentice's qualitative discovery, and show that both central temperature and ${ }^{8} \mathrm{~B}$ neutrino flux are reduced, with a minimum for a core mass of $0.025 M_{\odot}$; however, this minimum is only $20 \%$ less than the 'normal' flux of ${ }^{8} \mathrm{~B}$ neutrinos. Even so the results for idealised static models are still better than those obtained when an evolutionary sequence is calculated. A model evolved from a zero age situation in which the helium core was 0.025 $M_{\odot}$ reached one solar luminosity after $4.7 \times 10^{9} \mathrm{yr}$, when the ${ }^{8} \mathrm{~B}$ neutrino flux was only $1.5 \%$ below that for a 'standard' Sun evolved from a homogeneous zero age state. that of cytoplasmic mRNA. An observation now reported by Hirsch et al. (Cell, 1,$31 ; 1974)$ is that the size of the poly(A) fragment is virtually identical in human and insect mitochondria. Although no function has yet been suggested for the poly(A) in mitochondrial mRNA, its presence is clearly unrelated to transport and may be solely intramitochondrial, perhaps related to whatever function it has in the cytoplasmic messengers. Also of importance is the observation that the poly (A)-containing mitochondrial mRNA can be fractionated into some eight species, which together with the tRNA and rRNA sequences previously identified may account for transcription of the complete mitochondrial genome. These experiments suggest a view of the mitochondrion as an entity whose exolution has to some extent been independent of the surrounding cytoplasm.

Extending their previous studies to report now the first determination of the location of specific sequences within the non-messenger parts of nuclear HnRNA, Molloy, Jelinek, Salditt and Darnell (Cell, 1, 43; 1974) suggest a tentative structure for the large precursor. That the sequence destined to become mRNA lies at the $3^{\prime}$ end of the molecule to which poly $(\mathrm{A})$ is added is reasonably well established. But a crucial question concerns the composition of the remainder of the molecule, which is to be degraded. It is especially important to identify the junction between the mRNA and non-mRNA regions and to establish the criteria used by the maturation system for selecting the messenger.

The technique used by Molley et al. was to isolate poly(A)-containing HnRNA on poly(U) sepharose columns, break these molecules with alkali, and then reisolate the $3^{\prime}$ terminal sequences (which still contain poly(A)). By examining the properties of fragments of different lengths, it is possible to deduce the nature of the sequences located on the $5^{\prime}$ side of the messenger part of the molecule.

Because HnRNA can hybridise to DNA at rates more rapid than mRNA, it appears to contain transcripts of repetitive sequences absent from the messenger sequences. Using fragments isolated by the alkaline cleavage of HnRNA, Molloy et al. found that the first 3,000-4,000 $3^{\prime}$ nucleotides hybridise at the same rate as mRNA, but that as the fragment length increases to 8,000 nucleotides the rate increases $3-4$ times to the value characteristic of HnRNA. Since mRNA is in general about 3,000 nucleotides long, this result implies that the HnRNA molecule must contain repetitive sequences fairly near to the $5^{\prime}$ end of the mRNA section. Because the rate of hybridisation remains constant from a fragment length of $8,000-30,000$ bases, the rapidly hybridising regions in this part of the HnRNA may be about equally interspersed with less rapidly hybridising regions.

Another marker used to pin down specific parts of $\mathrm{HnRNA}$ is the presence of oligo(U) fragments, generally about thirty bases long. The poly(A) terminated HnRNA molecules larger than some 20,000 nucleotides possess about three oligo(U) fragments each; shorter HnRNAs possess a smaller proportion of these sequences; and mRNA lacks them altogether. More than $90 \%$ of the oligo(U) segments are located further than 12,000 nucleotides from the $3^{\prime}$ terminus and may be clustered at closely located sites.

A generalised structure for HnRNA thus locates mRNA and poly(A), occupying about 3,000 nucleotides, at the $3^{\prime}$ end. Pointing to the observation that rapid hybridisation of HnRNA depends on both cleavage and denaturation, Molloy et al. suggest that hairpin structures, in general less than 500 bases in length, may occupy the sequences up to about 15,000 nucleotides from the $3^{\prime}$ terminus. Sequences of oligo(U) may then be located towards the $5^{\prime}$ end of the HnRNA.

Whereas the addition of poly(A) seems to be a feature distinguishing the mRNA sequences of HnRNA which are to be conserved, a different mechanism has been known for some time to apply to ribosome RNA in eukaryotes. A large precursor, sedimenting at $45 \mathrm{~S}$ in mammalian cells, is methylated only in certain regions; and these regions ultimately become the $18 \mathrm{~S}$ and $28 \mathrm{~S}$ rRNAs. The rest of the molecule is degraded. Methylation has until now been thought to be a prerogative of rRNA and tRNA (in which it is necessary for the functions of the molecule) alone. But Perry and Kelley (Cell, 1, 37; 1974) now provide evidence that mRNA of mouse $L$ cells is also methylated. After labelling with ${ }^{3} \mathrm{H}$-methionine and ${ }^{14} \mathrm{C}$-uridine, the ${ }^{3} \mathrm{H} /{ }^{14} \mathrm{C}$ ratio of the poly $(\mathrm{A})$-containing RNA of the polysomes is about one fifth of that of rRNA. From this comparison, Perry and Kelley calculate that the proportion of methyl groups in mRNA is about $0.22 \%$. This means that an average mRNA of $3,000 \mathrm{nu}$ cleotides should bear six to seven methyl groups.

Both base and ribose moieties suffer methylation, the proportion of base methylation in mRNA being greater than in rRNA. A preliminary analysis suggested that there may be only one 